\title{
MicroRNA-126 functions as a tumor suppressor in colorectal cancer cells by targeting $\mathrm{CXCR} 4$ via the AKT and ERK1/2 signaling pathways
}

\author{
YALING LIU $^{1 *}$, YU ZHOU $^{2 *}$, XIAO FENG ${ }^{2}$, PING AN $^{1}$, XIAOJING QUAN ${ }^{1}$, HAO WANG $^{2}$, \\ SHICAI YE ${ }^{2}$, CAIYUAN YU ${ }^{2}$, YANTING $\mathrm{HE}^{2}$ and HESHENG LUO ${ }^{1}$
}

${ }^{1}$ Department of Gastroenterology, Renmin Hospital of Wuhan University, Wuhan, Hubei; ${ }^{2}$ Department of Gastroenterology, The Affiliated Hospital of Guangdong Medical College, Xiashan District, Zhanjiang, Guangdong, P.R. China

Received September 12, 2013; Accepted October 21, 2013

DOI: 10.3892/ijo.2013.2168

\begin{abstract}
Recent evidence shows that altered microRNA-126 (miR-126) expression is implicated in the progression of colorectal cancer (CRC). However, the precise roles and mechanisms of miR-126 in CRC remain unclear. The aim of this study was to investigate the roles of miR-126 in CRC cells and to elucidate miR-126-mediated mechanisms in CRC cells. First, miR-126 expression was analyzed using qRT-PCR in 4 human CRC cell lines (SW480, SW620, HT-29 and HCT-116). Furthermore, the biological properties of miR-126 in CRC cells in vitro were examined by applying Cell Counting Kit 8, cell cycle, cell apoptosis and transwell assays. The mechanisms and pathways of miR-126-mediated in CRC cells were detected by using qRT-PCR, western blotting and luciferase reporter assay. We found that miR-126 overexpression inhibited cell proliferation, migration and invasion, and induced cell arrest in the G0/ G1 phase of CRC cells, suggesting that miR-126 functions as a tumor suppressor in CRC cells. Furthermore, we identified the CXC chemokine receptor 4 (CXCR4) as a target of miR-126, and showed that it was negatively regulated by miR-126. We demonstrated that miR-126-mediated tumor suppression might be partly dependent on AKT and ERK1/2 signaling pathways. In conclusion, our data revealed that miR-126 functions as a tumor suppressor in CRC cells by regulating CXCR4 expression via the AKT and ERK1/2 signaling pathways and might be a novel target for therapeutic strategies in CRC.
\end{abstract}

Correspondence to: Professor Hesheng Luo, Department of Gastroenterology, Renmin Hospital of Wuhan University, 238 Jiefang Road, Wuhan 430060, Hubei, P.R. China

E-mail: xhnk@163.com

*Contributed equally

Key words: microRNA, miR-126, CXCR4, colorectal cancer, AKT, ERK1/2

\section{Introduction}

Colorectal cancer (CRC) is the third most common malignancy and the third leading cause of cancer-related mortality among men and women in the United States (1). It is estimated that there is a total of 142,820 new cancer cases diagnosed and 50,830 CRC deaths in 2013, accounting for $9 \%$ of all cancer deaths (1). Despite the improvement of multimodal anticancer strategies, the prognosis of advanced CRC is very poor, with 5 -year survival rates for stage III and stage IV colon cancer of 65.4 and $12.8 \%$, respectively (2). Thus, it is critically important to further explore the molecular pathogenesis of CRC, which may provide new targets for diagnosis and treatment.

MicroRNAs (miRs) are a type of non-coding, endogenous, small RNAs with a length of approximately 23 nucleotides that can regulate gene expression at the post-transcriptional level through binding to the 3' untranslated region (3'-UTR) of the target mRNA, subsequently triggering either translational repression or mRNA cleavage $(3,4)$. Growing evidence suggests that miRs are associated with a wide array of biological processes, including metabolism, development, cell proliferation, differentiation and apoptosis (5). To date, miR dysregulation has been described in numerous malignancies (6-9). These abnormal miRs are involved in cancer development, acting as tumor suppressors or oncogenes by negatively regulating their targets (10).

In this way, several miRs are involved in cell proliferation, migration, invasion, and poor disease prognosis of CRC, including miR-126. MiR-126 has been reported frequently to be downregulated in human cancers and may function as a tumor suppressor (11-13). It has been revealed that miR-126 targets IRS1, VEGF, p85 $\beta$, and some other molecules to inhibit cancer growth (14-16). Moreover, some studies have shown that miR-126 acts as negative regulator of tumor invasion and metastasis in cancers $(12,17,18)$. However, the precise roles and mechanisms of miR-126 in CRC remain to be elucidated.

In this study, we confirmed that miR-126 acts as a tumor suppressor in CRC cells, by inhibiting cell proliferation, migration, and invasion, and inducing G0/G1 phase arrest. Furthermore, we found that miR-126 targets CXC chemokine receptor 4 (CXCR4) and we explored the underlying mecha- 
nism of miR-126 in CRC, which may provide new therapeutic strategies for CRC.

\section{Materials and methods}

Cell culture and reagents. The CRC cell lines SW480, SW620, HT-29, and HCT-116 were routinely cultured in RPMI-1640 medium (Gibco, USA) supplemented with $10 \%$ fetal bovine serum, $100 \mathrm{U} / \mathrm{ml}$ penicillin, and $100 \mu \mathrm{g} / \mathrm{ml}$ streptomycin at $37^{\circ} \mathrm{C}$ in a humidified air atmosphere containing $5 \% \mathrm{CO}_{2}$. Exponentially growing cells were used for experiments. MiR-126 mimic, miR-126 negative control mimic (NC mimic), miR-126 inhibitor, and miR-126 negative control inhibitor (NC inhibitor) were purchased from Ribobio (RiboBio Co. Ltd., China).

Transfection of cell lines. SW480, SW620 or HT-29 cells were transfected with either miR-126 mimic (or NC mimic) $(50 \mathrm{nM})$ or miR-126 inhibitor (or NC inhibitor) $(100 \mathrm{nM})$, by using Lipofectamine 2000 (Invitrogen, USA) according to the manufacturer's protocol. At $48 \mathrm{~h}$ post-transfection, cells were collected for qRT-PCR, Cell Counting Kit 8 (CCK-8; Dojindo, Japan), cell apoptosis, and transwell assays. Western blot and cell cycle analyses were performed at $72 \mathrm{~h}$ after transfection.

qRT-PCR analysis of mRNA and miRNA expression. For miRNA and mRNA analyses, total RNA was isolated from cells using TRIzol reagent (Invitrogen). qRT-PCR was performed using SYBR $^{\circledR}$ Premix Ex Taq ${ }^{\text {TM }}$ II (Takara, Japan) according to the manufacturer's protocol. The expression of miR-126 and CXCR4 mRNA was normalized to U6 and $\beta$-actin, respectively. The $2^{-{ }^{-C T}}$ method was used for analysis. Forward miRNA and mRNA primers were synthesized by Sangon Biotech (Shanghai, China) and the sequences of the primers used were as follows: miR-126, 5'-tcgtaccgtgagtaataatgcg-3' (forward); U6, 5'-ctcgcttcggcagcaca-3' (forward); universal qRT-RCR primer, the One Step PrimeScript ${ }^{\circledR}$ miRNA cDNA Synthesis kit (Takara) (reverse); CXCR4, 5'-ttgtcatcctgtcctgctattg-3' (forward), 5'-tgttctcaaactcacaccettg-3' (reverse); $\beta$-actin, 5'-ggc ggcaacaccatgtaccet-3' (forward) and 5'-aggggecggactcgtcat act-3' (reverse).

Cell viability assay. A total of $3 \times 10^{3}$ SW480 and HT-29 cells per well were plated in 96-well plates for $24 \mathrm{~h}$ and then transfected with miR-126 mimic or NC mimic. Cell viability was measured at $48 \mathrm{~h}$ post-transfection using CCK8 assay according to the manufacturer's instructions. The absorbance was determined at $450 \mathrm{~nm}$.

Cell cycle and apoptosis assay. At 48 or $72 \mathrm{~h}$ post-transfection with miR-126 mimic or NC mimic, SW480 and HT-29 cells were trypsinized. For cell cycle assay, the cells were fixed with $70 \%$ ethanol at $4^{\circ} \mathrm{C}$ overnight, then treated with RNase A $(50 \mu \mathrm{g} / \mathrm{ml})$, stained with PI $(50 \mu \mathrm{g} / \mathrm{ml})$ (Beyotime, China) for $30 \mathrm{~min}$ and subjected to flow cytometry. For apoptosis assay, the cells were resuspended in binding buffer containing Annexin V-FITC and PI (Beyotime) according to the manufacturer's instructions. Apoptosis was analyzed by flow cytometry. Annexin V-FITC-positive and PI-negative cells were observed to undergo apoptosis.

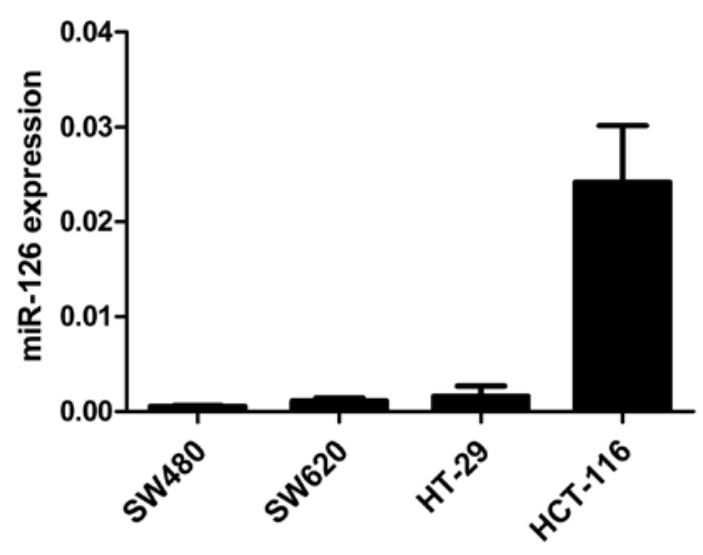

Figure 1. MiR-126 expression in CRC cell lines. MiR-126 expression measured by qRT-PCR. Values are presented as means \pm SD from 3 experiments.

Migration and invasion assay. SW480 and SW620 cells were used at $48 \mathrm{~h}$ post-transfection with miR-126 mimic or NC mimic. A 24 -well transwell plate $(8-\mu \mathrm{m}$ pore size; Corning Costar, USA) was used to measure migratory and invasive ability in vitro. For migration assay, $2 \times 10^{5}$ cells were plated in the top chamber with a non-coated membrane. For invasion assay, $3 \times 10^{5}$ cells were seeded in the top chamber coated with Matrigel (BD Biosciences, USA). In both assays, cells were suspended in serum-free medium, and the lower chamber was filled with RPMI-1640 containing $20 \%$ fetal bovine serum. After incubation at $37^{\circ} \mathrm{C}$ for $24 \mathrm{~h}$, non-migratory cells on the upper surface were removed using a cotton swab, then fixed in methanol and stained with $0.1 \%$ crystal violet (Beyotime). Five random fields were analyzed for each insert at x100 magnification under a microscope.

Construction of luciferase reporter vectors and luciferase reporter assay. DNA fragments of the 3'-UTRs of CXCR4 mRNA (RefSeq NM_001008540) containing the putative binding sites of miR-126 were synthesized. The fragments were then subcloned into the $\mathrm{XhoI}$ and NotI sites downstream of the Renilla luciferase coding region in the psiCHECK-2 vector (Promega, USA), and validated by sequencing by Sangon Biotech. The reporter plasmids with wild-type 3'-UTR or mutated 3'-UTR were named as PsiCHECK2-CXCR4-wt or PsiCHECK2-CXCR4-mut, respectively. SW480 cells were plated at $1 \times 10^{5}$ cells/well on 24-well plates and co-transfected with PsiCHECK2-CXCR4-3'-UTR (psi-CXCR4), PsiCHECK2-mutCXCR4-3'-UTR (psi-CXCR4-mut), or empty vector plasmid and miR using Lipofectamine 2000. Cells were harvested at $48 \mathrm{~h}$ post-transfection, and Firefly and Renilla luciferase activities were analyzed using the Dual-Luciferase Report assay (Promega) according to the manufacturer's instructions.

Western blotting. Total cellular proteins were lysed using RIPA buffer (Beyotime). Equivalent amounts of protein were resolved by $10 \%$ SDS-PACE gel, transferred onto PVDF membranes (Millipore, USA), and incubated with antibodies to CXCR4 (Abcam, UK), AKT (pan), phospho-AKT (Ser473), ERK1/2, or phospho-ERK1/2 (Cell Signaling Technology, USA) at $4^{\circ} \mathrm{C}$ overnight. The protein bands were then incubated with 

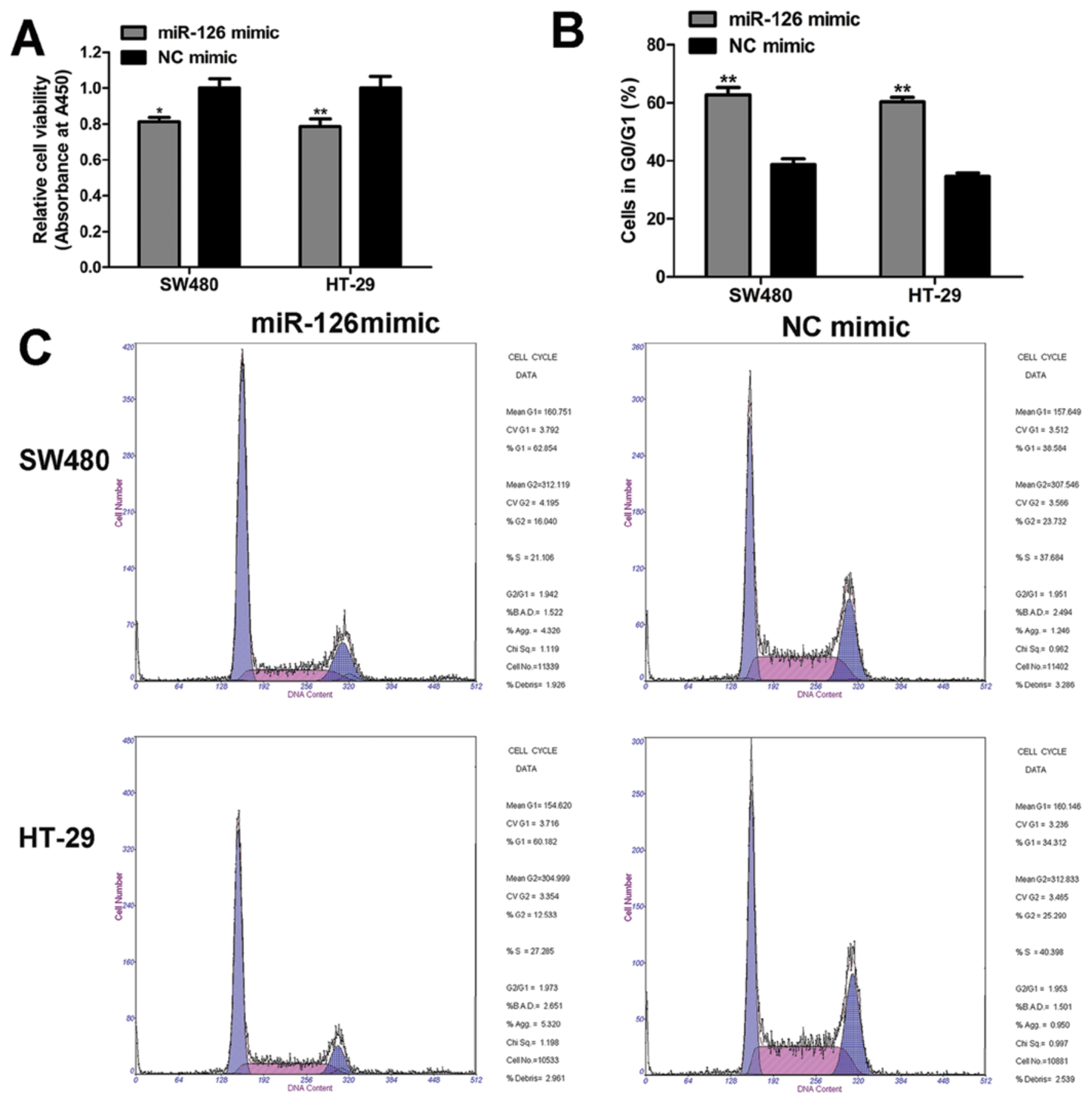

Figure 2. Effects of miR-126 on cell viability and cell cycle of CRC cells. (A) CCK8 assay. MiR-126 mimic transfection inhibited cell viability. (B) Average proportion of cells in G0/G1 phase. G0/G1 phase arrest was induced by miR-126 mimic. (C) Representative images of the cell cycle. Data are shown as the means \pm SD of 3 separate experiments $\left({ }^{*} \mathrm{P}<00.05,{ }^{* *} \mathrm{P}<0.01\right)$.

horseradish peroxidase-conjugated antibodies (Beyotime) and visualized using ECL. GADPH was used as a loading control.

Statistical analysis. All experiments were performed in triplicate. Data are expressed as the means \pm SD. Differences between groups were calculated with one-way ANOVA and Student's t-test. Differences were considered significant at $\mathrm{P}<0.05$.

\section{Results}

MiR-126 expression in CRC cell lines. qRT-PCR was performed to evaluate miR-126 levels in 4 CRC cell lines
(SW480, SW620, HT-29 and HCT-116). As shown in Fig. 1, miR-126 expression was downregulated in CRC cell lines and might be involved in CRC development.

MiR-126 inhibits cell proliferation, and induces G0/G1 arrest of CRC cells. To determine the roles of miR-126 in CRC cells, we adopted CCK8 assay, cell cycle, and apoptosis assay to evaluate the biological properties of miR-126. The results of CCK8 assay implied that cell viability was significantly impaired by miR-126 mimic as compared to NC mimic (Fig. 2A). In addition, in the cell cycle distribution analysis, we found that miR-126 overexpression enhanced the proportion of 
A
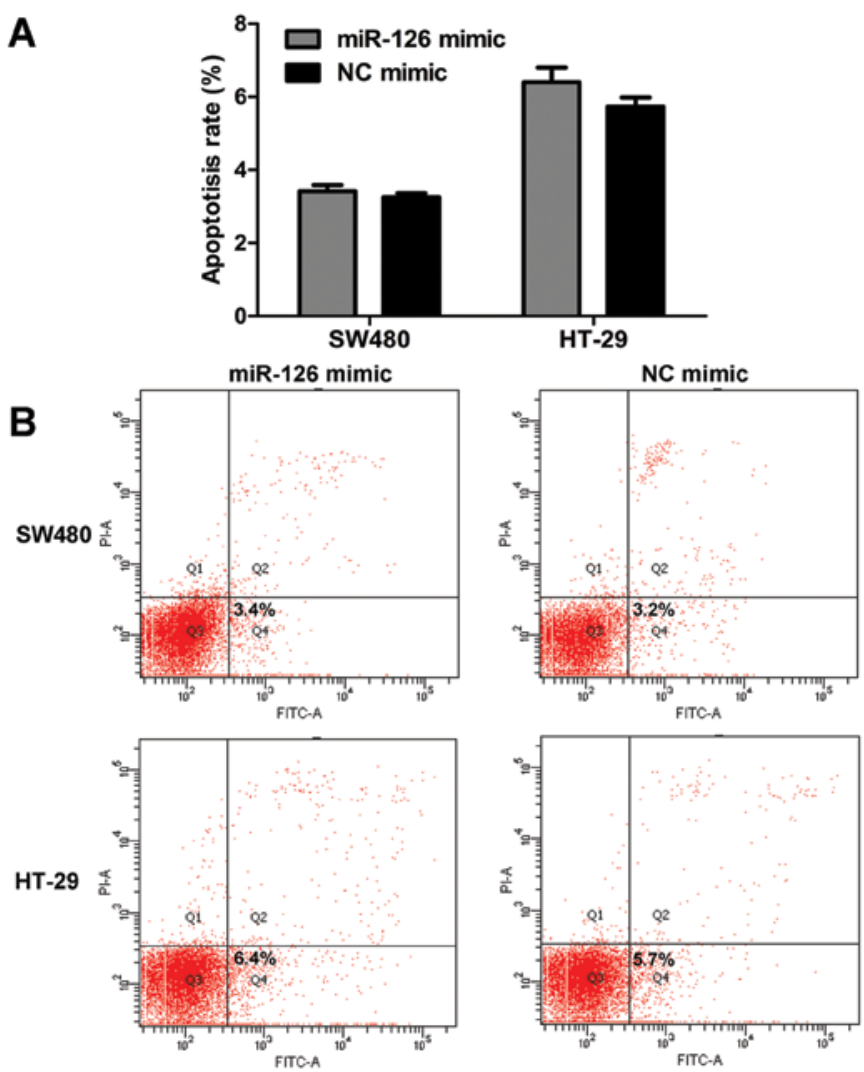

Figure 3. Effects of miR-126 on cell apoptosis of CRC cells. (A) Average apoptosis rate. MiR-126 mimic had no effect on cell apoptosis. (B) Representative images depicting cell apoptosis. Data are shown as the means \pm SD of 3 separate experiments. cells in the G0/G1 phase compared with NC mimic (Fig. 2B and C). However, miR-126 had little effect on the apoptosis rate (Fig. 3). Based on these data, we conclude that miR-126 mimic inhibits cell proliferation, and induces G0/G1 phase arrest, but does not affect the apoptosis of CRC cells.

MiR-126 suppresses cell migration and invasion of CRC cells in vitro. To elucidate the involvement of miR-126 in migration and invasion, the transwell assay was employed to evaluate the migration and invasion capacity of CRC cells. We found that miR-126 overexpression inhibited cell migration (Fig. 4A and $\mathrm{B})$. The results of the invasion assay revealed that the number of invaded cells was reduced when cells were treated with miR-126 mimic (Fig. 4C and D). Overall, these data show that miR-126 overexpression suppresses the migration and invasion capacity of CRC cells.

CXCR4 is a target of miR-126. By using bioinformatic analysis tools, i.e., MicroCosm Targets (http://www.ebi. ac.uk/enright-srv/microcosm/htdocs/targets/v5/) and PicTar (http://pictar.mdc-berlin.de/), we sought to predict a putative binding sites of miR-126 that are located at bases $239-245 \mathrm{nt}$ of the CXCR4 3'-UTR (Fig. 5A). To further confirm whether CXCR4 is a direct target of miR-126, the luciferase reporter vectors of the CXCR4 3'-UTR or its mutation containing miR-126 binding sites were constructed. Co-transfection was performed with miR-126 mimic (or NC mimic), psi-CXCR4 (or psi-CXCR4-mut, or empty vector) plasmids into SW480 cells, the dual-luciferase reporter assay was used to determine the reporter activities of the different constructs. We observed

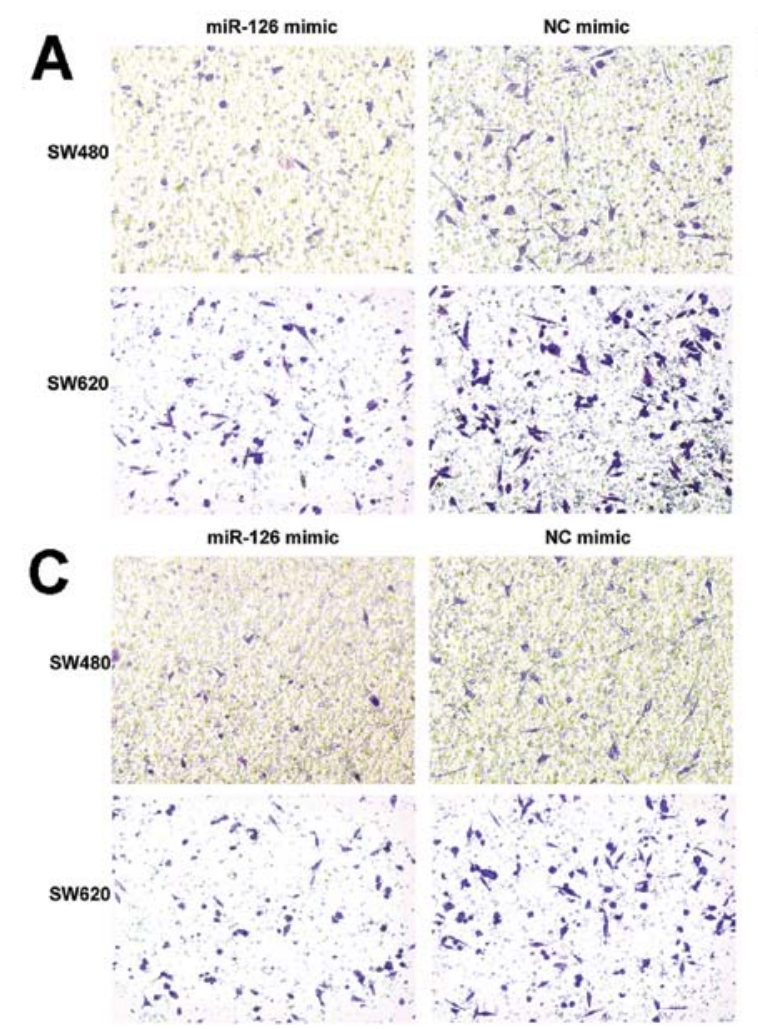

B

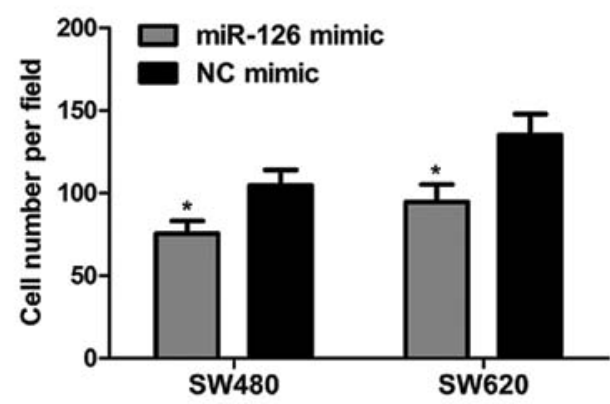

D

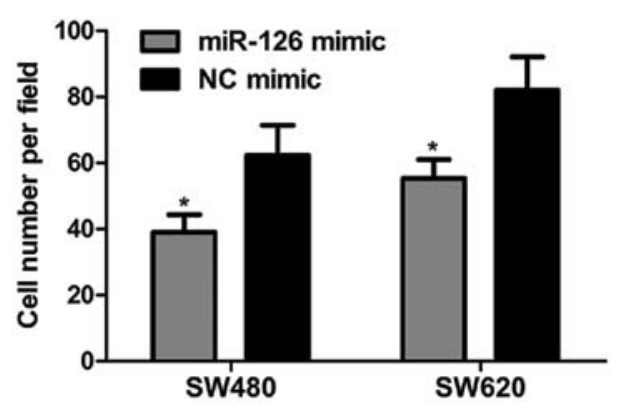

Figure 4. Effects of miR-126 on cell migration and invasion of CRC cells. (A) Migration assay. Representative images of migrated cells on the membrane (magnification, x100). (B) Quantification of migrated cells per field. MiR-126 mimic inhibited cell migration. (C) Invasion assay. Representative images of invaded cells on the membrane (magnification, x100). (D) Quantification of invaded cells per field. MiR-126 mimic inhibited cell invasion. Data are presented as the means \pm SD of 3 separate experiments $\left({ }^{*} \mathrm{P}<0.05\right)$. 

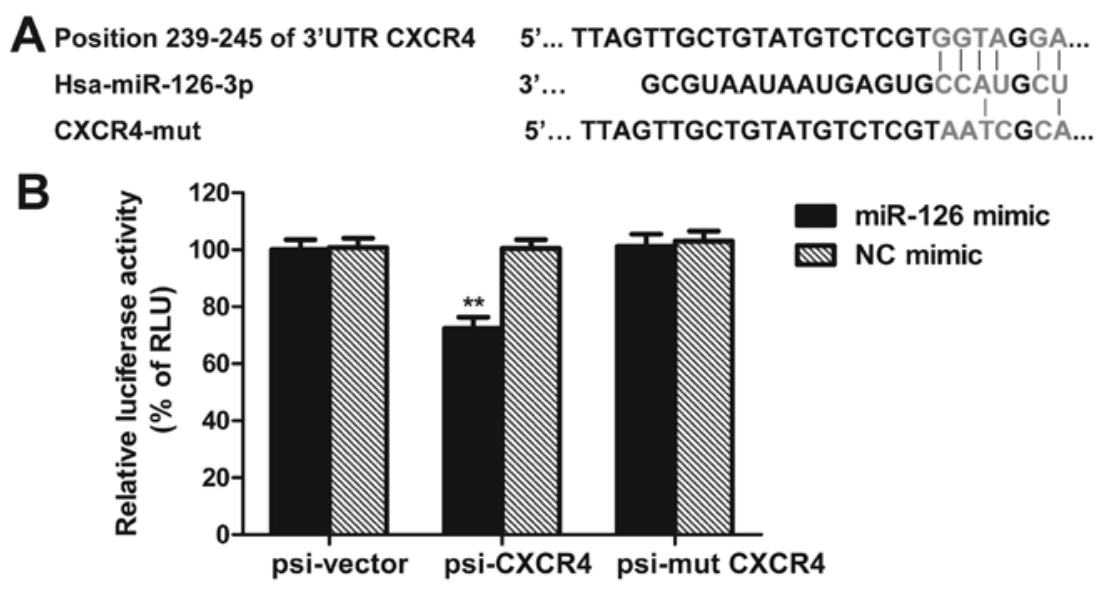

Figure 5. MiR-126 targets CXCR4. (A) Sequence alignment of predicted miR-126 binding sites within the CXCR4 3'-UTR and its mutation for luciferase reporter assay. (B) Luciferase reporter assay. Co-transfection with miR-126 mimic (or NC mimic) and psi-CXCR4 (or psi-CXCR4-mut or empty vector) plasmid into SW480 cells. Firefly luciferase activity was normalized to Renilla luciferase activity. Relative luciferase activities are presented. MiR-126 mimic repressed luciferase activities of psi-CXCR4. Data are presented as the means \pm SD of 3 measurements $\left({ }^{* *} \mathrm{P}<0.01\right.$, compared to that of psi-vector).
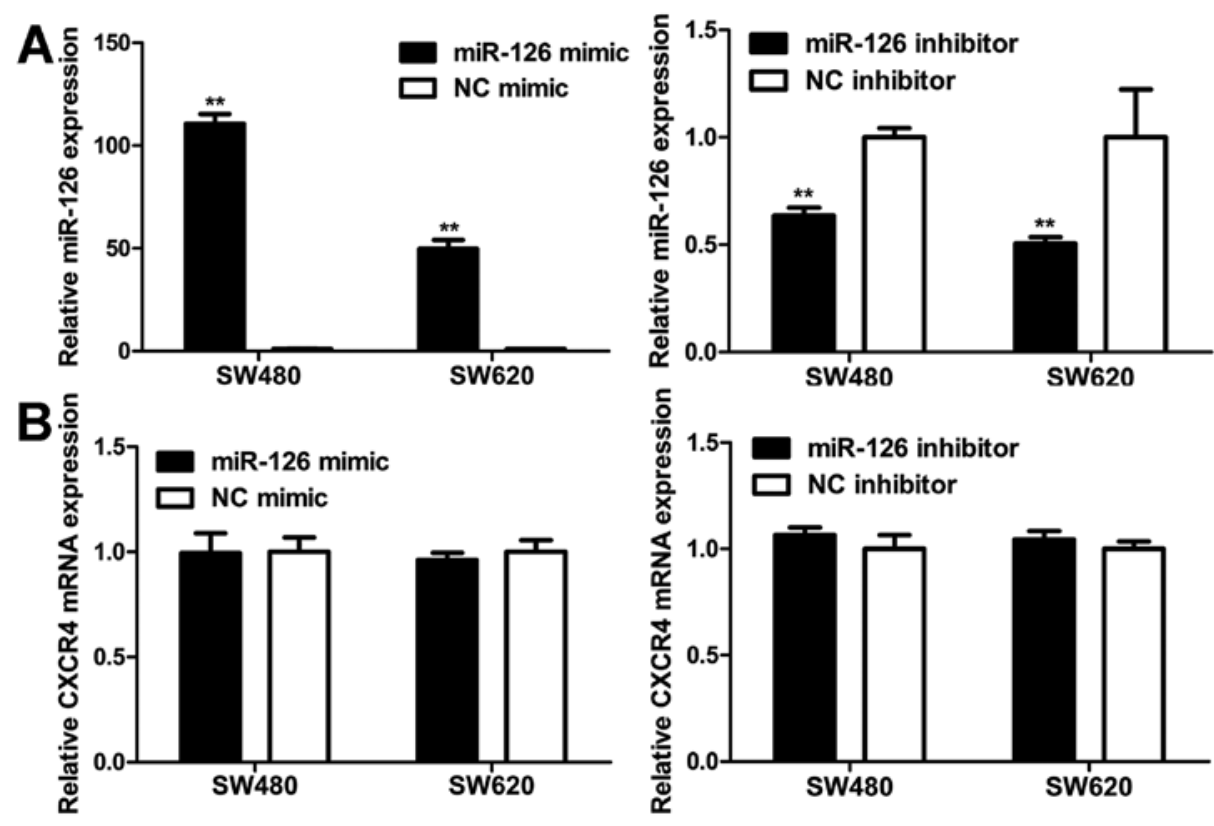

Figure 6. Regulation of CXCR4 mRNA by miR-126. (A) The relative miR-126 expression was assessed by qRT-PCR. Cells transfected with miR-126 mimic increased miR-126 expression, while cells transfected with miR-126 inhibitor showed a decrease in miR-126 expression. (B) The relative expression of CXCR4 mRNA. Transfected cells showed no changes in CXCR4 mRNA level. Data are shown as the means $\pm \mathrm{SD}$ of 3 separate experiments. $\left({ }^{* *} \mathrm{P}<0.01\right)$.

that the luciferase activity of psi-CXCR4 was repressed by miR-126 mimic as compared with control (Fig. 5B). Moreover, miR-126 mimic reduced CXCR4 protein level, while miR-126 inhibitor enhanced CXCR4 protein expression (Fig. 7). These results demonstrate that CXCR4 may be a direct target of miR-126.

MiR-126 negatively regulates CXCR4 expression at the posttranscriptional level. To explore the modulation of CXCR4 expression by miR-126, western blot and qRT-PCR analyses were carried out in SW480 and SW620 cells. MiR-126 level was markedly elevated in cells transfected with miR-126 mimic compared to NC mimic (Fig. 6A). Western blot analyses revealed that the increase in miR-126 expression resulted in a decrease in the CXCR4 protein level (Fig. 7). On the contrary, transfection with miR-126 inhibitor reduced miR-126 expression (Fig. 6A), which increased the CXCR4 protein level (Fig. 7). However, unlike the CXCR4 protein expression, CXCR4 mRNA level remained unaltered with regard to miR-126 level (Fig. 6B). Our results indicate that miR-126 negatively regulates CXCR4 expression at the posttranscriptional level.

Effects of miR-126 on AKT and ERK1/2 signaling pathways. To determine whether there were some changes in AKT and ERK1/2 signaling pathways in miR-126-transfected cells, we examined the phosphorylation status of AKT and ERK $1 / 2$ by western blotting. We found that miR-126 overexpression 

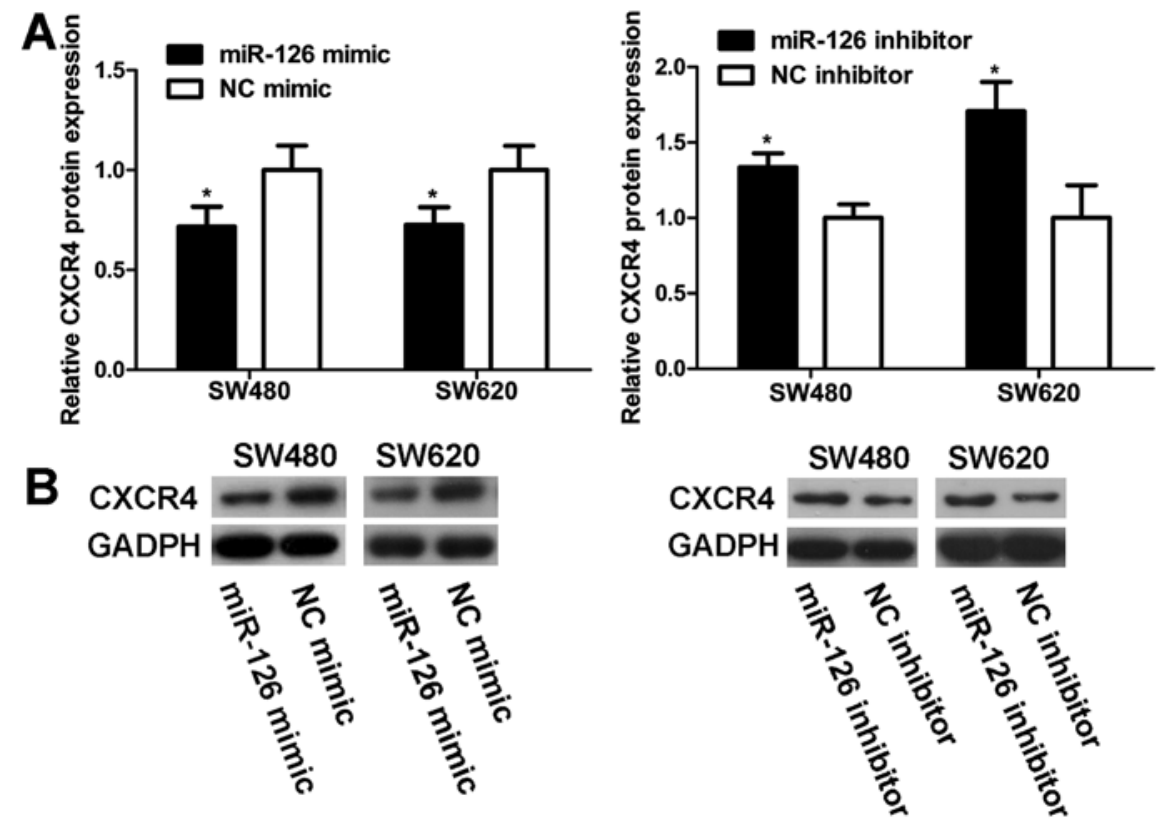

Figure 7. Regulation of CXCR4 protein by miR-126. (A) Quantification of the relative level of CXCR4 protein. Transfection with miR-126 mimic resulted in decreased CXCR4 protein levels, while transfection with miR-126 inhibitor increased CXCR4 protein levels, when compared with corresponding negative control. (B) Representative images of CXCR4 protein expression obtained by western blotting. Data are shown as the means \pm SD of 3 separate experiments $\left({ }^{*} \mathrm{P}<0.05\right)$.
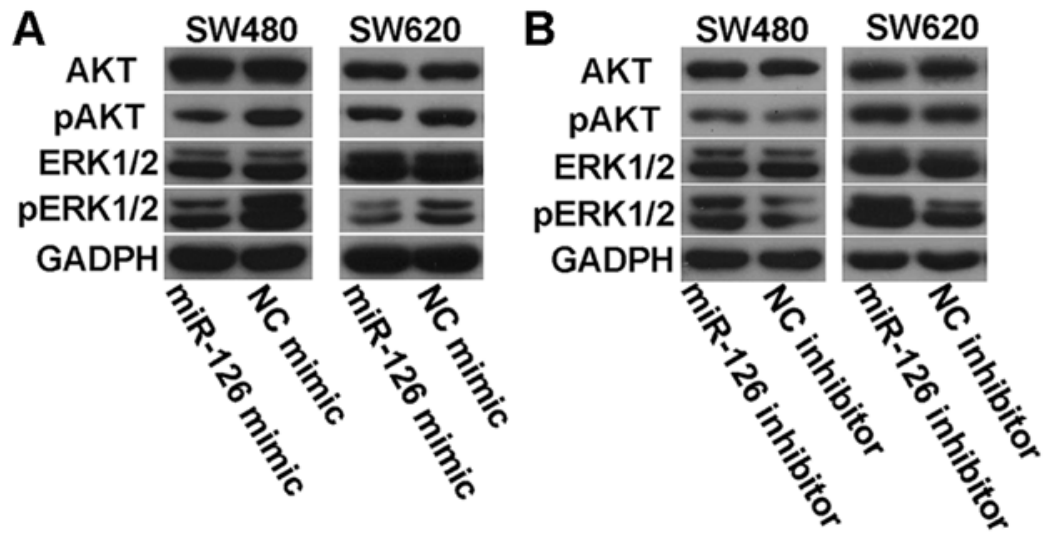

Figure 8. Effects of miR-126 on activation of AKT and ERK1/2 signaling pathways. MiR-126 mimic decreased the levels of pAKT and pERK1/2 (A), while miR-126 inhibitor promoted pAKT and pERK1/2 expression (B), without altering the levels of total-AKT and total-ERK1/2 protein, when compared with the corresponding negative control.

led to decreased levels of phosphorylated AKT and ERK1/2 (Fig. 8A), while transfection with miR-126 inhibitor elevated the expression of phosphorylated AKT and ERK1/2 (Fig. 8B). However, there was no difference in the levels of total AKT and ERK1/2 with regard to miR-126 (Fig. 8). These data suggest that miR-126 may be an important regulator in the AKT and ERK1/2 signaling pathways.

\section{Discussion}

Aberrant expression of miR-126 has been reported to be involved in human tumor development. However, the roles and mechanisms of miR-126 in CRC remain largely unknown. In this study, we focused on miR-126.
Previous studies have indicated that miR-126 is frequently downregulated in various cancers $(9,11-13)$. MiR-126 has also been shown to be downregulated in CRC $(11,14,19)$. Recently, accumulating evidence has demonstrated that miR-126 is a tumor-suppressive miRNA in several malignancies. MiR-126 has been shown to inhibit cancer cell proliferation and induce G0/G1 arrest by targeting IRS1, SLC7A5 and VEGF $(15,16,20)$. Moreover, miR-126 has been demonstrated to inhibit tumor invasion and metastasis in breast and pancreatic cancer $(12,17,18)$. MiR-126 has also been reported to suppress CRC cell growth, migration and invasion $(14,21)$ by regulating certain targets. According to these studies, miR-126 functions as a tumor suppressor in tumor development. However, it is still controversial as to whether miR-126 is a tumor suppressive or oncogenic miRNA. For instance, it was reported that miR-126 
inhibits apoptosis, increases the viability of AML cells, and enhances the colony-forming ability of mouse normal bone marrow progenitor cells alone through targeting Polo-like kinase 2 (22). Furthermore, some studies demonstrated that miR-126 contributes to angiogenesis by targeting Spred-1 and PIK3R2, which are negative regulators of the vascular endothelial growth factor pathway $(23,24)$. Moreover, miR-126 was found to inhibit SOX2 expression and further contribute to gastric carcinogenesis (25). In view of these different reports, it is indispensable to further investigate the roles of miR-126 in regulating the biological properties of CRC cells in vitro.

In this study, we found that endogenous miR-126 expression was downregulated in CRC cell lines, implying that miR-126 may participate in CRC carcinogenesis. In addition, our results showed that miR-126 overexpression suppressed cell proliferation, and induced cell cycle arrest in G0/G1 phase, but did not affect apoptosis of CRC cells. Furthermore, we confirmed that restoration of miR-126 expression could weaken migration and invasion capability of CRC cells in vitro. These observations are in agreement with most previous reports, suggesting that miR-126 acts as a tumor suppressor in CRC cells and is a potential target for the therapeutics intervention of CRC.

To clarify possible mechanisms of miR-126-mediated tumor-suppression in CRC cells, we performed bioinformatics analyses to identify candidate target genes of miR-126. Due to the crucial roles of CXCR4 in human cancers, CXCR4 was chosen as a potential target of miR-126 for further research. CXCR4, the receptor for chemokine CXCL12 (26), is known to be widely expressed in various types of human cancers, including CRC. Several studies have established that CXCR4 plays a key role in tumor initiation, progression, metastasis and survival in CRC $(27,28)$. However, how CXCR4 mediates these processes has not been elucidated clearly. It has previously been shown that CXCR4 expression was regulated by some miRNAs, e.g., by miR-146a in Kaposi's sarcoma-associated herpesvirus (29), by miR-150 in bone marrow-derived mononuclear cells (30), and by miR-146a to control megakaryopoiesis (31). We demonstrated that CXCR4 protein expression decreased in CRC cells by transfection with miR-126 mimic, and increased by transfection with miR-126 inhibitor. In contrast, CXCR4 mRNA levels in CRC cells were unaltered with regard to miR-126 expression. These findings suggest a miR-126-mediated post-transcriptional regulatory mechanism. Moreover, we confirmed that CXCR4 might be a direct target of miR-126 by dual-luciferase reporter assay. From these data, we conclude that CXCR4 is negatively regulated by miR-126, which may explain, at least in part, the tumorsuppressive effects of miR-126 in CRC cells. Certainly, there are other potential mechanisms accounting for miR-126-mediated tumor suppression of CRC, e.g., other targets of miR-126 and other regulators of miR-126. Furthermore, the regulation of CXCR4 expression by miR-126 might not be as prominent as some other mechanisms in CRC.

It has been suggested that AKT and ERK are downstream targets of CXCR4 signal transduction $(32,33)$. Shen et al showed that CXCR4-induced proliferation was mediated by AKT and ERK signaling in human pancreatic cancer cells (34). Brand et al reported that CXCR4-mediated homing and CRC cell migration may be highly dependent on AKT and ERK signaling (35). On the basis of these reports, we wanted to find out whether miR-126-mediated tumor suppression depends on AKT or ERK1/2 signaling pathways via regulating CXCR4 expression. As expected, miR-126 restoration reduced the expression of phosphorylated AKT and ERK1/2, whereas miR-126 inhibitor increased the levels of phosphorylated AKT and ERK1/2, without altering total AKT and ERK1/2 protein levels. These data indicate that miR-126 might be an important regulator in AKT and ERK1/2 signaling. Moreover, it is possible that modulation of the CXCR4 expression by miR-126 resulted in the subsequent regulation of AKT and ERK1/2 phosphorylation. Hence, we propose that miR-126-induced tumor suppression may be mediated by modulation of CXCR4 via AKT and ERK1/2 signaling pathways. However, the complex mechanisms of miR-126-mediated tumor suppression need to be further elucidated.

In conclusion, our data suggest that miR-126 functions as a tumor suppressor in CRC cells, by inhibiting cell proliferation, migration, and invasion and inducing cell arrest in the G0/G1 phase, but not by affecting cell apoptosis. Moreover, miR-126-mediated tumor suppression may, in part, depend on the regulation of CXCR4 via AKT and ERK1/2 signaling pathways. Therefore, miR-126 could be a diagnostic as well as therapeutic target for CRC therapy.

\section{References}

1. Siegel R, Naishadham D and Jemal A: Cancer statistics, 2013. CA Cancer J Clin 63: 11-30, 2013.

2. Lan YT, Yang SH, Chang SC, et al: Analysis of the seventh edition of American Joint Committee on colon cancer staging. Int J Colorectal Dis 27: 657-663, 2012.

3. Bartel DP: MicroRNAs: target recognition and regulatory functions. Cell 136: 215-233, 2009.

4. Pasquinelli AE: MicroRNAs and their targets: recognition, regulation and an emerging reciprocal relationship. Nat Rev Genet 13: 271-282, 2012.

5. Bartel DP: MicroRNAs: genomics, biogenesis, mechanism, and function. Cell 116: 281-297, 2004.

6. Asangani IA, Rasheed SA, Nikolova DA, et al: MicroRNA-21 (miR-21) post-transcriptionally downregulates tumor suppressor Pdcd 4 and stimulates invasion, intravasation and metastasis in colorectal cancer. Oncogene 27: 2128-2136, 2008.

7. Ozen M, Creighton CJ, Ozdemir M and Ittmann M: Widespread deregulation of microRNA expression in human prostate cancer. Oncogene 27: 1788-1793, 2008

8. Calin GA and Croce CM: MicroRNA signatures in human cancers. Nat Rev Cancer 6: 857-866, 2006.

9. Wang X, Tang S, Le SY, et al: Aberrant expression of oncogenic and tumor-suppressive microRNAs in cervical cancer is required for cancer cell growth. PLoS One 3: e2557, 2008.

10. Babashah S and Soleimani M: The oncogenic and tumour suppressive roles of microRNAs in cancer and apoptosis. Eur $\mathrm{J}$ Cancer 47: 1127-1137, 2011.

11. Li XM, Wang AM, Zhang J and Yi H: Down-regulation of miR-126 expression in colorectal cancer and its clinical significance. Med Oncol 28: 1054-1057, 2011.

12. Tavazoie SF, Alarcon C, Oskarsson T, et al: Endogenous human microRNAs that suppress breast cancer metastasis. Nature 451: 147-152, 2008.

13. Feng R, Chen $\mathrm{X}, \mathrm{Yu} \mathrm{Y}$, et al: miR-126 functions as a tumour suppressor in human gastric cancer. Cancer Lett 298: 50-63, 2010.

14. Guo C, Sah JF, Beard L, Willson JK, Markowitz SD and Guda K: The noncoding RNA, miR-126, suppresses the growth of neoplastic cells by targeting phosphatidylinositol 3-kinase signaling and is frequently lost in colon cancers. Genes Chromosomes Cancer 47: 939-946, 2008.

15. Liu B, Peng XC, Zheng XL, Wang J and Qin YW: MiR-126 restoration down-regulate VEGF and inhibit the growth of lung cancer cell lines in vitro and in vivo. Lung Cancer 66: 169-175, 2009. 
16. Zhang J, Du YY, Lin YF, et al: The cell growth suppressor, mir-126, targets IRS-1. Biochem Biophys Res Commun 377: $136-140,2008$.

17. Hamada S, Satoh K, Fujibuchi W, et al: MiR-126 acts as a tumor suppressor in pancreatic cancer cells via the regulation of ADAM9. Mol Cancer Res 10: 3-10, 2012.

18. Zhang Y, Yang P, Sun T, et al: miR-126 and miR-126* repress recruitment of mesenchymal stem cells and inflammatory monocytes to inhibit breast cancer metastasis. Nat Cell Biol 15: 284-294, 2013.

19. Zhang Y, Wang X, Xu B, et al: Epigenetic silencing of miR-126 contributes to tumor invasion and angiogenesis in colorectal cancer. Oncol Rep 30: 1976-1984, 2013.

20. Miko E, Margitai Z, Czimmerer Z, et al: miR-126 inhibits proliferation of small cell lung cancer cells by targeting SLC7A5. FEBS Lett 585: 1191-1196, 2011.

21. Li N, Tang A, Huang S, et al: MiR-126 suppresses colon cancer cell proliferation and invasion via inhibiting RhoA/ROCK signaling pathway. Mol Cell Biochem 380: 107-119, 2013.

22. Li Z, Lu J, Sun M, et al: Distinct microRNA expression profiles in acute myeloid leukemia with common translocations. Proc Natl Acad Sci USA 105: 15535-15540, 2008.

23. Fish JE, Santoro MM, Morton SU, et al: miR-126 regulates angiogenic signaling and vascular integrity. Dev Cell 15 $272-284,2008$

24. Wang S, Aurora AB, Johnson BA, et al: The endothelial-specific microRNA miR-126 governs vascular integrity and angiogenesis. Dev Cell 15: 261-271, 2008.

25. Otsubo T, Akiyama Y, Hashimoto Y, Shimada S, Goto K and Yuasa Y: MicroRNA-126 inhibits SOX2 expression and contributes to gastric carcinogenesis. PLoS One 6: e16617, 2011.

26. Oberlin E, Amara A, Bachelerie F, et al: The CXC chemokine SDF-1 is the ligand for LESTR/fusin and prevents infection by T-cell-line-adapted HIV-1. Nature 382: 833-835, 1996.
27. Kim J, Takeuchi H, Lam ST, et al: Chemokine receptor CXCR4 expression in colorectal cancer patients increases the risk for recurrence and for poor survival. J Clin Oncol 23: 2744-2753, 2005.

28. Murakami T, Kawada K, Iwamoto M, et al: The role of CXCR3 and CXCR4 in colorectal cancer metastasis. Int J Cancer 132: 276-287, 2013.

29. Punj V, Matta H, Schamus S, Tamewitz A, Anyang B and Chaudhary PM: Kaposi's sarcoma-associated herpesvirusencoded viral FLICE inhibitory protein (vFLIP) K13 suppresses CXCR4 expression by upregulating miR-146a. Oncogene 29: 1835-1844, 2010

30. Tano N, Kim HW and Ashraf M: microRNA-150 regulates mobilization and migration of bone marrow-derived mononuclear cells by targeting Cxcr4. PLoS One 6: e23114, 2011.

31. Labbaye C, Spinello I, Quaranta MT, et al: A three-step pathway comprising PLZF/miR-146a/CXCR4 controls megakaryopoiesis. Nat Cell Biol 10: 788-801, 2008.

32. Yasumoto K, Koizumi K, Kawashima A, et al: Role of the CXCL12/CXCR4 axis in peritoneal carcinomatosis of gastric cancer. Cancer Res 66: 2181-2187, 2006.

33. Wendt MK, Drury LJ, Vongsa RA and Dwinell MB: Constitutive CXCL12 expression induces anoikis in colorectal carcinoma cells. Gastroenterology 135: 508-517, 2008.

34. Shen X, Artinyan A, Jackson D, Thomas RM, Lowy AM and Kim J: Chemokine receptor CXCR4 enhances proliferation in pancreatic cancer cells through AKT and ERK dependent pathways. Pancreas 39: 81-87, 2010.

35. Brand S, Dambacher J, Beigel F, et al: CXCR4 and CXCL12 are inversely expressed in colorectal cancer cells and modulate cancer cell migration, invasion and MMP-9 activation. Exp Cell Res 310: 117-130, 2005. 